Revista lus et Praxis, Año 18, No 2, 2012, pp. 219 - 240

ISSN 0717 - 2877

Universidad de Talca - Facultad de Ciencias Jurídicas y Sociales

"La consistencia en el lenguaje de las normas:

Hacia una propuesta consistente"

Fernando Centenera Sánchez-Seco

\title{
LA CONSISTENCIA EN EL LENGUAJE DE LAS NORMAS: HACIA UNA PROPUESTA CONSISTENTE*
}

\author{
CONSISTENCY IN REGULATORY LANGUAGE: \\ TOWARDS A CONSISTENT PROPOSAL
}

\begin{abstract}
Fernando Centenera Sánchez-Seco**
RESUMEN

La recomendación de consistencia en el lenguaje normativo puede encontrarse en guías o manuales de redacción, y también en varios estudios. Sin embargo, una revisión desde el punto de vista de la práctica invita a formular propuestas para obtener una recomendación más funcional. En este trabajo se ofrecen algunas ideas al respecto. En él se estudia la acepción de consistencia que pudiera ser más óptima y sus tipos, y se plantea la pregunta de si la recomendación ha de aplicarse en todo caso. Tomando como base esta exposición se propone una nueva formulación de la recomendación.

ABSTRACT

The recommendation that regulatory language should be consistent is common in guides or manuals for drafting as well as in several studies. Yet a review from the angle of practice demonstrates the desirability of a more functional recommendation. This study offers some ideas in this regard. It analyses which might be the optimal definition for consistency and its types, and it asks whether the recommendation should be applied in all cases. That discussion then serves as the basis for a new formulation of the recommendation.

PALABRAS CLAVE

Consistencia, Directrices de Técnica Normativa, Prudencia, Personal de Redacción

KEYWORDS

Consistency, Legal Drafting Guidelines, Prudence, Writing Staff
\end{abstract}

\section{INTRODUCCIÓN}

La preocupación por la calidad del lenguaje de las normas es un aspecto que podría considerarse tradicional en el ámbito anglosajón. Sin embargo, en

\footnotetext{
"Trabajo llevado a cabo en el marco del Proyecto de investigación "Neutro, punto de vista y traducción sexuada: de la teoría a las estrategias". Ministerio de Ciencia e Innovación. Ref. FEM2009-10976. El autor agradece al profesor Virgilio Zapatero por todos sus consejos y apreciaciones sobre este trabajo. Trabajo recibido el 23 de abril y aprobado el 7 de junio de 2012.

** Doctor en Derecho. Profesor de Filosofía del Derecho Universidad Alcalá de Henares, España. Correo electrónico: fernando.centenera@uah.es.
} 
las décadas más recientes la cuestión está solicitando también el interés en otros ámbitos. Quizá una prueba de ello sea la publicación cada vez más visible de guías y manuales de redacción normativa. En estos documentos se recogen listas más o menos amplias de recomendaciones lingüísticas. Resulta interesante constatar también que la producción de estudios dedicados al lenguaje normativo cada vez es más intensa. Todo ello evidencia que el tema en cuestión está generando interés, y que la intención de conseguir mejoras en este ámbito probablemente esté más presente que nunca. Sin embargo, también es cierto que al menos en determinadas ocasiones, estas aportaciones parecen tener un valor simbólico; algo que, ciertamente, no ha de ser despreciado, pero que no resulta suficiente si se adopta la óptica de la funcionalidad. Parece que si nos detenemos en el ámbito de la práctica y somos conscientes de las circunstancias a tener en cuenta en esta parcela, tendríamos que reformular numerosas recomendaciones.

En este trabajo nos proponemos asumir precisamente ese compromiso con la práctica, fijando la atención en una recomendación concreta en el contexto español: la consistencia. Es cierto que ésta puede apreciarse en las directrices de técnica normativa actuales y en trabajos de diferente tipo, pero a nuestro entender todavía sigue vigente la pregunta de si es posible seguir mejorando, con vistas a la consecución de propuestas más funcionales. Para responderla pensamos que, en primer lugar, conviene determinar qué queremos decir con la palabra consistencia, considerar los problemas que pueden surgir en el caso de que no se observe, y buscar en base a todo ello la acepción que se crea más conveniente. Nuestro desarrollo comenzará con la exposición de estos puntos, y con el bosquejo de una posible clasificación de consistencia. Sin embargo, el objetivo de buscar una fórmula lo más apta posible para la práctica invita también a que nos cuestionemos acerca de si en todo caso debe seguirse la directriz de consistencia. La respuesta a estos interrogantes y el desarrollo que la precede nos descubre una propuesta que matiza de forma importante la que actualmente encontramos en las directrices de técnica normativa, y por ello en el último punto de este trabajo analizamos cuáles podrían ser las fórmulas más convenientes, para llevar a cabo una posible adaptación de la propuesta esbozada.

\section{ACEPCIONES Y TIPOS DE CONSISTENCIA}

Podemos acercarnos a la cuestión de la consistencia centrando la atención en la Nomografía o el arte de redactar leyes de Bentham, editada por el Centro de Estudios Constitucionales (utilizamos la segunda edición de 2004). En esta obra se considera la "incertidumbre en la expresión", dentro de las imperfecciones de segundo orden. Ésta tiene lugar cuando en la redacción de normas 
encontramos frases o palabras diferentes, que hacen referencia a un mismo significado. Bentham alerta acerca de la necesidad de evitar esta circunstancia, y ello por varias razones. Por una parte se refiere a la posible oscuridad que puede suscitar. El hecho de que encontremos expresiones diferentes, como poco puede inducir en la persona que lee la norma la sospecha de que con ellas se está haciendo referencia a significados diferentes ${ }^{1}$. Si este es el caso la utilización de expresiones diferentes sería apropiada, pero no en el supuesto de que con ellas se estuviera haciendo alusión a una misma idea. Fijémonos en el siguiente extracto:

"f) Establecer un sistema integral de tutela institucional en el que la Administración General del Estado, a través de la Delegación Especial del Gobierno contra la Violencia sobre la Mujer, en colaboración con el Observatorio Estatal de la Violencia sobre la Mujer, impulse la creación de políticas públicas dirigidas a ofrecer tutela a las víctimas de la violencia contemplada en la presente Ley.

g) Fortalecer el marco penal y procesal vigente para asegurar una protección integral, desde las instancias jurisdiccionales, a las víctimas de violencia de género" ${ }^{\prime 2}$.

Nos encontramos ante dos puntos de un artículo en el que se establecen diferentes medidas. Como puede observarse, en el primero de ellos se utiliza la expresión "violencia contemplada en la presente Ley", y en el segundo "violencia de género". En este caso si ambas expresiones hicieran referencia a dos ideas diferentes, la utilización de dos expresiones sería la apropiada, pero no acontecería del mismo modo si estuvieran haciendo alusión a una misma idea. A nuestro entender esta última circunstancia se aprecia en el caso que nos ocupa. Como es sabido, la Ley de Violencia de Género, de donde hemos extraído el extracto, tiene como objeto un tipo de violencia de género que no contempla todos los casos de este tipo de violencia, sino aquellos en los que la violencia se lleva a cabo contra la esposa del autor, o mujer que esté o haya estado vinculada al mismo por una relación de afectividad, aun sin existir convivencia. $\mathrm{Si}$, como hemos interpretado, las dos expresiones en cuestión se estuvieran refiriendo a la idea recientemente expuesta, tal circunstancia sería cuestionable desde el punto de vista de la consistencia, por cuanto podría inducir a pensar en la consideración de tipos de violencia diferente.

Es cierto que quizá en numerosas ocasiones no sea complejo el proceso que lleve a la solución de las posibles dudas que puedan surgir en los supuestos

\footnotetext{
${ }^{1}$ Bentham, Jeremy, Nomografía o el arte de redactar leyes, Centro de Estudios Políticos y Constitucionales, Madrid, 2004, p. 35.

${ }^{2}$ Ley Orgánica № 1/2004, de 28 de diciembre, de Medidas de Protección Integral contra la Violencia de Género, Boletín Oficial del Estado № 313, Miércoles 29 diciembre 2004, p. 42169.
} 
que nos ocupan, pero incluso aunque fuera así, la ausencia de consistencia probablemente habrá fomentado la distracción de quien lee la norma ${ }^{3}$. Por otra parte, Bentham nos dice que la circunstancia que consideramos puede ser también una vía que alimente la voluminosidad del texto. Imaginemos que en una norma se expresa un mismo significado con dos palabras o expresiones, una más voluminosa que la otra. En tales supuestos resultaría contraproducente para la economía del lenguaje utilizar la expresión voluminosa, cuando podrían alcanzarse los mismos objetivos con una fórmula más breve. Por todo ello, se recomienda que si lo que se pretende es hacer referencia a dos o más cosas diferentes, deben utilizarse expresiones diferentes ${ }^{4}$. A sensu contrario deberá entenderse que si se quiere hacer referencia a una única cosa, conviene que se utilice una única expresión. Serían, por tanto, contrarios a la recomendación de consistencia ejemplos como el siguiente: "La actividad que se limita pura y simplemente al mero desempeño del cargo..." ${ }^{5}$. Como puede observarse, en esta ocasión se utilizan tres palabras 'pura', 'simplemente' y 'mero' para hacer referencia a una misma idea, la de exclusividad. Curiosamente, si recurrimos al Diccionario de la Real Academia Española y buscamos la palabra 'mero', descubriremos que una de sus acepciones, la primera y la que parece ajustarse más al extracto trascrito, hace referencia a los adjetivos 'puro' y 'simple'

El legado de Bentham ha sido recogido posteriormente en numerosos manuales y guías de redacción en diferentes contextos, bajo el nombre de consistencia o coherencia lingüística. En estas referencias se recomienda utilizar el mismo término para expresar la misma idea ${ }^{7}$. Las directrices de técnica normativa españolas no son una excepción. Siquiera de forma colateral, en ellas se considera el tema de la consistencia al comienzo del documento, cuando se hace alusión a las directrices que aparecieron en 1991, y se señala que aquellas permitieron

\footnotetext{
${ }^{3}$ Strunk, William y White, E. B., The elements of style, Allyn and Bacon, third edition, Boston, 1979, p. 80. La apreciación hace referencia a los símiles, pero entendemos que puede ser aplicable a nuestro caso.

${ }^{4}$ Bentham, Nomografía, cit. nota n. 1, pp. 19, 35, 36.

${ }^{5}$ Ley 20/2007, de 11 de julio, del Estatuto del trabajo autónomo, Boletín Oficial del Estado № 166, Jueves 12 julio 2007, p. 29968.

${ }^{6}$ REAL ACADEMIA ESPAÑOLA, Diccionario de la Lengua Española, [s. e.], [s. I.], 2001. En: http://buscon. rae.es/drael/ [visitado el 02/01/12].

7 Vid. v. gr. TEXAS LEGISLATIVE COUNCIL, Drafting Manual, [s. e.], [s. I.], 2011. En: http://www. tlc.state.tx.us/legal/dm/sec723.htm [visitado el 08/03/12]; MARTINO, Antonio, Manual de Técnica Legislativa, [s. e.], [s. I.], [s. d.]. En http://exwww.dsp.unipi.it/didattica/Digesto/manual.html [visitado el 01/12/11]; GRUPPO DI LAVORO PER LA REVISIONE DEL MANUALE REGIONALE DI DRAFTING, Regole e suggerimenti per la redazione dei testi normativi, G. Tamara Tarabusi e Massimo Signorile del Consiglio regionale della Toscana, Terza edizione, Toscana, 2007. En: http://lexview-int.regione. fvg.it/iterleggi/drafting/drafting.pdf [visto el 08/03/12].
} 
homogeneizar técnicamente los textos ${ }^{8}$. Lo cierto es que esta apreciación puede resultar significativa, pero debe decirse que no es especialmente representativa para este trabajo, dado que en aquellas directrices de 1991 no se había incluido el apartado de criterios lingüísticos que sí recogen las de 2005. No obstante, hay que decir que en este último documento localizamos otra referencia más explícita cuando en él se establecen recomendaciones sobre los términos técnicos, donde se considera que han de utilizarse en todo el documento con igual sentido 9 . Conviene, no obstante, reparar en el dato de que en esta ocasión, la recomendación se concreta en un aspecto determinado de la redacción: los términos técnicos. De otro lado, en el mismo documento podemos localizar el siguiente extracto: "Se evitará... la españolización de términos extranjeros cuando en nuestro idioma tienen otro significado, y es conveniente mantener una terminología unitaria a lo largo del texto"10 ${ }^{\prime 1}$. Ciertamente, en este punto la redacción del texto dista de ser la deseada. En ella parece darse a entender que podría haber supuestos en los que no debería seguirse la recomendación de consistencia (si se considera "...cuando [...] es conveniente mantener una terminología unitaria...", habrá que entender que en determinadas ocasiones no será conveniente dicho ejercicio). Ello, en principio no tendría por qué descartarse, como más adelante se verá, pero sin duda este aspecto no aparece convenientemente justificado en el texto de las directrices. Por otra parte, también en esta cuestión nos encontramos con que la cuestión se aborda de forma parcial, por cuanto alcanza únicamente a aspectos terminológicos.

Sin duda, las directrices de técnica normativa de 2005 representan un paso adelante en la preocupación por conseguir un lenguaje normativo de mayor calidad. Prueba de ello es el hecho de que en ellas se haya incluido las referencias recientemente expuestas. Sin embargo, ello no obsta para seguir pensando en la mejora. Dejando a un lado los problemas de oscuridad que se desprenden de la lectura del documento, debe decirse que si lo que se pretende es reducir en lo posible los problemas a los que hacía referencia Bentham, parece que será conveniente considerar una acepción de consistencia, capaz no ya sólo de alcanzar a determinados aspectos terminológicos, ni incluso a la terminología en

\footnotetext{
${ }^{8}$ Resolución de 28 de julio de 2005, de la Subsecretaría, por la que se da publicidad al Acuerdo del Consejo de Ministros, de 22 de julio de 2005, por el que se aprueban las Directrices de técnica normativa, Boletín Oficial del Estado № 180, Viernes 29 julio 2005, p. 26878.

${ }^{9}$ Resolución de 28 de julio de $2005 \ldots$... cit. nota 8, p. 26888.

${ }^{10}$ Resolución de 28 de julio de $2005 \ldots$, cit. nota 8 , p. 26888. Lo cierto es que a nuestro modo de ver, no resulta fácil interpretar el extracto en el que se inserta la cuestión que nos ocupa. Se trata del siguiente texto: "Se evitará el uso de extranjerismos cuando se disponga de un equivalente en casteIlano, la utilización de palabras y construcciones lingüísticas inusuales, así como la españolización de términos extranjeros cuando en nuestro idioma tienen otro significado, y es conveniente mantener un [sic.] terminología a lo largo del texto".
} 
su conjunto, sino también a otros aspectos de la redacción en los que no parece nada desdeñable la recomendación de consistencia. Las propuestas de Bentham se han ido dejando notar también a lo largo de los siglos en numerosos trabajos, que hoy ya se consideran clásicos en el mundo de la redacción normativa. Pues bien, en algunas de estas referencias se recoge el tipo de acepción que venimos buscando. Pensamos que una de las aportaciones más representativas es la que ofrece Dickerson, que considera que una misma idea ha de expresarse de la misma forma, e ideas diferentes de diferente forma ${ }^{11}$. Interesante es también la obra de Butt y Castle, donde se considera lo que denominan una segunda golden rule enunciada como sigue: nunca debe cambiarse el lenguaje a menos que se desee cambiar su significado. Con ello se promueve un estilo claro, directo y conciso $^{12}$. Como puede apreciarse, estas fórmulas ya no sólo alcanzan a aspectos terminológicos, sino también, aunque de modo implícito, a otros aspectos, como, por ejemplo, los números, las mayúsculas, las enumeraciones, las fechas, los signos de moneda, etc. A nuestro juicio esta formulación general es más completa, porque siendo la que más ámbitos abarca, también será la que ofrezca menos oportunidades para que aparezcan problemas como los diagnosticados anteriormente en diferentes ámbitos. De otro lado, puede señalarse también que una formulación enunciada en términos más concretos (que se centre, por ejemplo, en los términos técnicos), al menos desde una interpretación literal podría dar lugar a entender que la cuestión de la consistencia se considera recomendable en los casos establecidos, pero no en los demás.

Es cierto que en la práctica, la recomendación de consistencia traerá consigo la repetición de palabras, expresiones, etc. Éste, sin embargo, no debe considerarse un problema del lenguaje jurídico. De hecho, en numerosas referencias aparece como recomendación, y se aconseja al tiempo no sustituir el término o expresión repetida por pronombres personales o adjetivos demostrativos ${ }^{13}$. La variación elegante no es elegante en el lenguaje jurídico ${ }^{14}$.

\footnotetext{
${ }^{11}$ DiCKERSON, Reed, The Fundamentals of Legal Drafting, Little, Brown and Company, Boston 1986, p. 15.

${ }^{12}$ Butt, Peter and CASTLE, Richard, Modern Legal Drafting. A Guide to Using Clearer Language, Cambridge University Press, Cambridge, 2001, pp. 48, 49.

${ }^{13}$ Martino, Manual, cit. nota n. 7; GRUPPO DI LAVORO PER LA REVISIONE DEL MANUALE REGIONALE DI DRAFTING, Regole, cit. nota n. 7, p. 28; FUNDACIÓN HUMANISMO POLÍTICO A. C., Manual de Técnica Legislativa, [s. e.], [s. I.], [s. d.]. En http://www.agora-parl.org/sites/default/files/ FHP\%20-\%20Manual\%20de\%20Tecnica\%20legislativa\%20-\%20ES\%20-\%20PI.pdf [visto el 08/03/12]; López Ruiz, Miguel, Redacción Legislativa, Senado de la República, México, 2002, pp. 70-72. En http:// www.senado.gob.mx/biblioteca/content/catalogos/b_digital/bibliodigital/redaccionlegislativa.pdf [visto el 19/07/10].

${ }^{14}$ Sáinz Moreno, Fernando, "Problemas actuales de técnica normativa", Anuario jurídico de La Rioja, № 1, 1995, p. 62; BenION, Francis, On Statute Law, Logman, [s. I.], 1990, p. 189. En http://www.fran-
} 
Además de todo lo dicho, conviene señalar también que es posible establecer una clasificación de tipos de consistencia. Es probable que a medida que se ha ido leyendo el desarrollo precedente, se haya podido estar pensando en la aplicación de las recomendaciones en cuestión en el texto de una norma. Ciertamente, ésta es una posible forma de concebir la consistencia, pero no la única. En trabajos de diferente tipo la recomendación se hace extensible a aquellos textos normativos con los cuales existe relación con respecto la norma que se redacta ${ }^{15}$. Desde esta perspectiva se entiende que las normas que regulen una determinada cuestión, deberían guardar la consistencia en su lenguaje; incluso la recomendación se extiende a la legislación vigente en general en determinados trabajos. A propósito de ello se ha diferenciado entre coherencia interna, que hace referencia al desarrollo del párrafo precedente, y coherencia externa, que comprende los supuestos recientemente expuestos $^{16}$.

\section{3. ¿LA CONSISTENCIA COMO REGLA UNIVERSAL?}

Un repaso de los trabajos, guías y manuales de redacción normativa nos descubre que en muchas ocasiones, la recomendación de consistencia se enuncia con carácter universal. Tras la lectura de tales recomendaciones da la impresión de que lo que se está solicitando es que, en todo caso, el equipo de redacción vele por la consistencia en los textos que redacta. Cabe, sin embargo, preguntarse si este ejercicio es siempre el más apropiado. Quizá en un principio sintamos inclinación por una respuesta afirmativa, prima facie probablemente resulte complicado encontrar motivos que cuestionen el hecho de redactar un texto normativo de forma consistente. Sin embargo, un análisis más detenido y comprometido con la práctica, ofrece algunas razones para poner en cuestión esta primera percepción.

cisbennion.com/pdfs/fb/1990/1990-002-187-statute-law-pt2-ch12.pdf [visto el 16/08/10]; CONNATSER, Bradford R., "Two centuries in progress in technical communication", Technical writing and communication Vol. 37 (2), 2007, pp. 137, 138; Azuelos-AtiAs, Sol, "On the incoherence of Legal Language to the General Public", International Journal for the Semiotics of Law, June, 2010. En http://www. springerlink.com/content/d072m75556167j35/ [visto el 16/08/10]; SeIDMAN, Ann, SeIDMAN, Robert B., AbeYESEKERE, Nalin, Legislative Drafting for Democratic Social Change. A Manual for Drafters, Kluwer Law International, London, 2001, pp. 264 y 265.

15 Peces-Barba Martínez, Gregorio, Diez lecciones sobre Ética, Poder y Derecho, Dykinson, Madrid, 2010, p. 322; MARTINO, Manual, cit. nota n. 7; GRUPPO DI LAVORO PER LA REVISIONE DEL maNuAle ReGiOnale Di DRAFtinG, Regole, cit. nota n. 7, p. 28; Seidman, Seidman, Abeyesekere, Legislative, cit. nota n. 14, p. 265.

16 MeSEGUer YeBRA, Joaquín, Guía práctica para la elaboración de textos normativos. Directrices estatales de técnica normativa, Boch, Barcelona, 2008, p. 26. 
Aristóteles, en su Ética a Nicómaco, distingue entre la ciencia y la fronesis o prudencia. Sobre la primera dice que aquello de lo que tenemos ciencia no puede ser de otra manera, que el objeto de la ciencia es necesario y, por tanto, imperecedero. Sin embargo, cuando se refiere a la fronesis o prudencia considera que en ella sí tiene lugar la discusión sobre lo bueno. En esta ocasión ya no nos encontramos ante algo que no puede ser de otra manera o que necesariamente debe darse ${ }^{17}$. Se ha dicho que es precisamente la perspectiva de la prudencia aristotélica, la que conviene adoptar a la hora de analizar los aspectos relativos a la redacción de las normas ${ }^{18}$. A nuestro juicio la reflexión es acertada, y sirve perfectamente para introducir las siguientes reflexiones con las que pretendemos cuestionar el supuesto carácter universal de la consistencia.

Quizá una buena forma de comenzar el desarrollo en este espacio sea refiriéndonos a la Constitución y teoría general de la interpretación jurídica de Wróblewski. En esta obra se establece que si hay razón suficiente, es posible atribuir el mismo significado a términos diferentes ${ }^{19}$. A nuestro juicio, en esta afirmación se encuentra la idea de que la consistencia no ha de observarse en todo caso, dado que en determinadas ocasiones razones de peso podrían justificar una opción diferente. Pero, ¿qué razones podrían considerarse? Pensamos que algunas de las más relevantes podrían enmarcarse, en primer lugar, dentro de los objetivos de la efectividad normativa y, en segundo lugar, dentro de los objetivos de la eficacia normativa ${ }^{20}$. Como es sabido, el fin general de la legislación es dirigir la conducta de las personas a las que se destinan las normas, orientar sus comportamientos en un sentido determinado ${ }^{21}$; en definitiva, conseguir normas que sean eficaces. Pero entre los fines de la legislación y con mayor relevancia, también se considera la efectividad, que hace referencia a la capacidad de las normas para conseguir los objetivos a los que se dirige ${ }^{22}$, y que constituye el punto capital de cualquier teoría de la legislación que pretenda un compromiso con el derecho como medio de dirección social ${ }^{23}$. Ello nos remite al análisis de las

\footnotetext{
${ }^{17}$ ArISTÓteles, Ética a Nicómaco, Centro de Estudios Políticos y Constitucionales, Madrid, 1999, pp. 91 y 92.

${ }^{18}$ XANTHAKI, Helen, "Drafting manuals and quality in legislation: positive contribution towards certainty in the law or impediment to the necessity for dynamism of rules?", Legisprudence. International Journal for the Study of Legislation, Vol. IV, 2, 2010, p. 111.

${ }^{19}$ WRÓBLEWSkı, Jerzy, Constitución y teoría general de la interpretación jurídica, Civitas, Madrid, 1985, p. 48.

${ }^{20}$ Zapatero, Virgilio, El Arte de Legislar, Aranzadi, Pamplona, 2009, p. 33.

${ }^{21}$ Zapatero, El Arte, cit. nota n. 20, p. 33; Marcilla Córdoba, Gema, Racionalidad legislativa. Crisis de la ley y nueva ciencia de la legislación, Centro de Estudios Constitucionales, Madrid, 2005, p. 287.

22 Zapatero, El Arte, cit. nota n. 20, p. 33; Xanthaki, "Drafting", cit. nota n. 18, p. 114.

${ }^{23}$ Zapatero, El Arte, cit. nota n. 20, p. 34.
} 
políticas públicas que tratan de implementarse con las normas ${ }^{24}$. En los próximos párrafos vamos a considerar algunos supuestos en los que, a nuestro entender, pueden percibirse de forma más concreta algunas de las razones que pudieran encuadrarse dentro de los objetivos expuestos y que, como hemos dicho, pueden servir para sustentar la postura expuesta.

En numerosas ocasiones se critica el lenguaje jurídico en general, señalando que es demasiado complejo, y que a menudo no es accesible a la ciudadanía. Ciertamente, al menos en nuestros días el objetivo de hacer normas comprensibles para todas las personas puede calificarse de muy lejano y también incluso de impreciso. Por una parte, en numerosas ocasiones el lenguaje de las normas ha de ser obligadamente complejo, y ello por razones de seguridad ${ }^{25}$. Por otro lado, la solicitud de hacer normas entendibles para toda la ciudadanía resulta cuestionable, por cuanto no en todo caso la ciudadanía en general es destinataria directa de las normas ${ }^{26}$; el tema de la complejidad de la audiencia es imposible de representar con un esquema tan simple. Sin embargo, todo ello no obsta para reconocer que el lenguaje normativo es sin duda susceptible de mejora, y que en ocasiones la forma en que se presenta puede dar lugar a problemas de diferente tipo ${ }^{27}$. Así se viene reconociendo desde hace tiempo en el ámbito anglosajón, principalmente por el cauce del Plain English, un movimiento que cada vez de forma más patente, se está dejando notar en contextos diferentes. Probablemente bajo la influencia de esta circunstancia, aunque no exclusivamente, en el ámbito académico español encontramos referencias en las que se aprecia una intención clara de mejorar el lenguaje normativo ${ }^{28}$. Es más, como bien sabemos el Consejo de Ministros ha aprobado dos acuerdos con la intención de mejorar la calidad de las normas; siendo especialmente representativo el de 2005, dado que incluye un apartado dedicado a los criterios lingüísticos.

\footnotetext{
${ }^{24}$ Zapatero, El Arte, cit., nota n. 20, p. 367.

${ }^{25}$ LAPORTA, Francisco J., "Imperio de la ley y seguridad jurídica", en Estado, justicia, derechos, Alianza Editorial, primera reimpresión, Madrid, 2011, pp. 112 y 113.
}

${ }^{26}$ Zapatero, El Arte, cit. nota n. 20, p. 267; Galiana Saura, Ángeles, La legislación en el Estado de Derecho, Dykinson, Madrid, 2003, p. 92.

${ }^{27}$ Quizá resulte interesante la consulta de KelLy, David, "The victorian experience of plain drafting", en Legislation and its interpretation. Discussion and seminar papers, Law Commission, Wellington, 1988, p. 58. En esta referencia se consideran una serie de problemas que acarreó la redacción de una serie de documentos (indemnizaciones -awards-), escritos de forma tan extremadamente legalista, que no los entendían ni los/as trabajadores/as a quienes iban dirigidos, ni el personal de dirección. Unos documentos que habían nacido con vocación de evitar disputas en el ámbito industrial, fueron origen de disputas, no sobre su contenido, sino sobre sus palabras y significados.

${ }^{28}$ Penamos que así acontece, por ejemplo, en Zapatero, El Arte, cit. nota n. 20; Prieto De Pedro, Jesús, Lenguas, lenguaje y derecho, Civitas, Madrid, 1991; Cazorla Prieto, Luis María, El Lenguaje Jurídico Actual, Aranzadi, Pamplona, 2007. 
Representativo es además que en los últimos meses se haya conformado una comisión, con el fin de redactar un informe, hoy ya publicado ${ }^{29}$, para la modernización del lenguaje jurídico.

Parece, por tanto, que el lenguaje normativo es susceptible de mejora, y que además las propuestas en torno a este objetivo están proliferando cada vez más. Tal circunstancia sin duda ha de considerarse de forma positiva, por cuanto con ella se persigue aportar grados más altos de seguridad para las personas destinatarias de las normas, al tiempo que cotas superiores de eficacia normativa. Sin embargo, al menos en parte esta proliferación de manifestaciones que postulan un lenguaje normativo mejor, hace que la recomendación general de consistencia se tambalee. Así acontece si, por ejemplo, centramos nuestro análisis en la coherencia externa que, como sabemos, hace referencia al hecho de que en las normas que regulan una misma cuestión, o en la legislación vigente en general, se utilice una misma forma para hacer alusión a una misma idea. Si esto es así, y si se concibe la recomendación de consistencia como aplicable en todo caso, se estaría recomendando, no sólo perpetuar los aspectos cuestionables del lenguaje de las normas jurídicas vigentes, sino también la imposibilidad de seguir guías, manuales o informes publicados con posterioridad a aquellas normas, con los que se pretende mejorar la calidad del lenguaje normativo. Quizá, a propósito de estas cuestiones, sea interesante referirnos a un trabajo de Laws, en el que se pone de manifiesto el dilema de si deben realizarse innovaciones y desarrollos que mejoran la calidad del lenguaje normativo, aun sabiendo que ello irá en detrimento de la consistencia, si se tiene en cuenta el lenguaje utilizado en las normas precedentes ${ }^{30}$. Ciertamente, en casos como éste la evaluación de riesgos es capital, y es en este punto donde la prudencia aristotélica debe entrar en juego. Ello, no obstante, no obsta para seguir manteniendo que la consistencia puede quedar cuestionada. El ejercicio de evaluación de riesgos puede inclinar la balanza a favor de la consistencia, pero nada impide pensar que el test de riesgos pueda también favorecer en determinadas circunstancias la inclinación contraria.

Parece, por tanto, que la propuesta de consistencia sin excepciones podría quedar cuestionada, por cuanto su seguimiento no haría posible seguir avanzando en la consecución de un lenguaje normativo mejor, con todas las consecuencias que ello pudiera conllevar. Pero eso no es todo. La intención

\footnotetext{
${ }^{29}$ INFORME DE LA COMISIÓN DE MODERNIZACIÓN DEL LENGUAJE JURÍDICO, 2011. En: http://portal.uned.es/pls/portal/docs/PAGE/UNED_MAIN/LAUNIVERSIDAD/VICERRECTORADOS/SECRETARIA/ NORMATIVA/RECOMENDACIONES_DE_LA_COMISI\%C3\%93N_DE_MODERNIZACI\%C3\%93N_ DEL_LENGUAJE_JUR\%C3\%8DDICO.PDF [visto el 23/01/12].

${ }^{30}$ Laws CB, Stephen, "Drawing the Line", en Drafting Legislation. A Modern Approach, Ashgate, England, 2008, p. 28.
} 
de plantear la recomendación de consistencia de forma universal, daría lugar a supuestos bastante paradójicos, en los que aquella pretensión conllevaría, incluso, la negación de la propia consistencia en algún sentido. La reflexión necesita explicación. Consideremos el siguiente supuesto. De un lado, pensemos en una norma en cuyo texto se utiliza un emparejamiento de sinónimos para hacer referencia a una idea. De otro lado, pensemos en otra norma posterior, donde se hace referencia a esa misma idea con el mismo emparejamiento de sinónimos. Con este ejercicio se preserva la consistencia, porque se utiliza una forma determinada para hacer referencia a una misma idea; pero al tiempo también se está cuestionando la consistencia, por cuanto el emparejamiento de sinónimos conlleva el hecho de utilizar varias palabras para hacer referencia a una misma idea, y ello, como ya vimos, es contrario a lo que se establece desde el punto de vista de la consistencia ${ }^{31}$.

No obstante, la intención de mejorar la calidad del lenguaje y las paradojas que pudieran seguirse de una concepción universal de la recomendación, no son las únicas circunstancias que podrían considerarse, a la hora de cuestionar la aplicación de la recomendación en todo caso. Para introducir la siguiente objeción vamos a referirnos a algunas apreciaciones que se han considerado en el ámbito de la redacción de normas, a propósito de la precisión y la claridad en su lenguaje. Se ha dicho que desde el Gobierno se espera que sus políticas tengan efecto legal, expresándose la intención legislativa de forma tan precisa como sea posible, siendo posible una única interpretación ${ }^{32}$. Sin embargo, lo que, en definitiva, debe buscarse es la consecución de los objetivos que se persiguen con la norma. Aunque ello pueda considerarse extraño en un principio $^{33}$, no parece que la afirmación anterior pueda generalizarse ${ }^{34}$. Con ello queremos decir que un proyecto que exprese las intenciones del Gobierno, no necesariamente ha de ser claro y preciso (piénsese, por ejemplo, en los casos en los que el texto normativo es oscuro, a consecuencia de dificultades para llegar a una acuerdo en el plano político ${ }^{35}$. Pues bien, a nuestro entender este planteamiento puede ser aplicable también a la cuestión que tratamos: en ocasiones, por diferentes razones, puede ser que para llevar a cabo determinados objetivos se precise de un texto normativo que no siga la

\footnotetext{
31 Zapatero, El Arte, cit. nota n. 20, pp. 258, 259; Peces-Barba Martínez, Diez, cit. nota n. 15, p. 322; Seidman, Seidman, Abeyesekere, Legislative, cit. nota n. 14, p. 265.

32 Crabbe, Vcrac, "The ethics of legislative drafting", Commonwealt law bulletin, Vol. 36, 1, March 2010, p. 16. Puede consultarse además ZaPATERo, El Arte, cit. nota n. 20, pp. 362, 363.

33 ZAPATERO, El Arte, cit. nota n. 20, p. 266.

${ }^{34}$ Miers, David R. and PAGe, Alan C., Legislation, Sweet \& Maxwell, London, 1982, p. 87.

${ }^{35}$ ZAPATERO, El Arte, cit. nota n. 20, p. 262; MIERS and PAGE, Legislation, cit. nota n. 34, pp. 90 y 91.
} 
recomendación de consistencia. A propósito de esta cuestión, quizá resulte ilustrativo centrarnos en la aplicación paulatina del lenguaje no sexista en diferentes leyes (especialmente representativas son las que abordan la igualdad) ${ }^{36}$. Con esta práctica no se promociona en numerosas ocasiones la consistencia. Sirva como ejemplo decir que para hacer referencia a la persona que ocupa una presidencia, se utilizan expresiones como "quien ostente la presidencia", "presidente o presidenta", etc. Si embargo, el ejercicio en cuestión puede estar justificado si desde el ámbito de las políticas públicas se entiende que de este modo se favorecerá la eficacia de la norma, o si con ello se pretende llevar a cabo una labor de concienciación acerca de la visibilidad de las mujeres en el lenguaje. Todo ello, no obstante, no impide señalar que también en estos casos, conviene llevar a cabo un análisis de posibles riesgos. Sería sin duda contraproducente que la seguridad se viera seriamente afectada por estas circunstancias.

Los análisis sobre consistencia en el ámbito que consideramos no concluyen con las consideraciones precedentes. Dando un paso más podría afirmarse que, aunque resulte paradójico, el test de seguridad al que antes nos referíamos en el párrafo precedente, podría a su vez comprometer la consistencia de una norma que se redacta con un lenguaje no sexista, precisamente a consecuencia de preservar la consistencia, no de la norma que se redacta, sino de otra previa. Tal circunstancia queda ejemplificada en aquellos casos en los que una norma que utiliza usos no sexistas del lenguaje, modifica o hace referencia a textos anteriores. En tales supuestos, ¿deben redactarse las modificaciones conforme a los usos no sexistas del lenguaje, promocionando así la consistencia de la norma que modifica (y que se escribe conforme a un lenguaje no sexista), o deben redactarse sin tales prácticas, promocionando en tal caso la consistencia de la norma modificada? Lo cierto es que la respuesta a este dilema no es fácil, y no se está resolviendo en la actualidad conforme a un criterio uniforme. Sin embargo, quizá resulte interesante señalar que desde el ámbito doctrinal anglosajón se ha recomendado dejar inalterado el texto de la norma precedente en cuanto a la utilización de usos no sexistas ${ }^{37}$. De nuevo en esta ocasión, por razones de seguridad o eficacia normativa, la recomendación de consistencia concebida en un sentido universal queda cuestionada.

\footnotetext{
${ }^{36}$ Sobre la trayectoria que se está siguiendo en relación a esta cuestión, puede verse CentenerA SÁnCHEZSECO, Fernando, Nombradas. También en el ámbito jurídico, Dykinson, Madrid, 2010.

${ }^{37}$ Christopher, Williams, "The End of the 'Masculine Rule'? Gender-Neutral Legislative Drafting in the United Kingdom and Ireland", Statute Law Review, 29 (3), pp. 139-153; TEXAS LEGISLATIVE COUNCIL, Drafting, cit. nota n. 7, p. 103; Centenera SÁnChez-SeCO, Fernando, "Una aproximación a la aplicación parcial del lenguaje neutral en la norma", Cuadernos Electrónicos de Filosofía del Derecho, № 18, 2009, p. 111. En http://ojs.uv.es/index.php/CEFD/article/view/129/141 [visto el 10/04/12].
} 
El esquema recientemente expuesto no es exclusivo del ejercicio de aplicar el lenguaje no sexista en las normas. En ocasiones, determinadas expectativas políticas justificadas pueden poner en cuestión la consistencia por diferentes razones. Pensemos, por ejemplo, en la Ley de Violencia de Género Estatal, con la que se crearon los juzgados de violencia de género. Según nos parece, en esta norma se trató probablemente de dar una especial relevancia a quienes trabajan con el derecho en los casos de violencia de género, y quizá por ello su denominación profesional aparece escrita con mayúscula inicial "Abogado", "Jueces" o "Secretarios Judiciales", por ejemplo) ${ }^{38}$. Sin embargo, esta rutina se interrumpe en determinadas partes de la norma. Así, en la disposición final sexta de la ley, donde la palabra "abogado" figura en minúscula ${ }^{39}$. Al menos en nuestra opinión, también en esta ocasión la falta de consistencia se debe a la intención de preservar la consistencia, no de la ley en cuestión, sino de la que queda modificada con la disposición final sexta (si buscásemos el razonamiento de todo ello, de nuevo nuestra reflexión desembocaría en la cuestión de preservar la seguridad o eficacia de la norma más antigua). El texto que modifica la Ley de Violencia de Género pertenece al párrafo 5 del artículo $3^{\circ}$ de la Ley 1/1996, de 10 de enero, de Asistencia Jurídica Gratuita ${ }^{40}$, una norma en la que, según hemos podido observar, la palabra "abogado" se escribe con minúscula inicial a lo largo del texto.

\section{HaCia una PROPUESTA CONSISTENTE}

A lo largo de este trabajo hemos ido considerando varios aspectos que, a nuestro juicio, deben tenerse en cuenta, si se pretende bosquejar una recomendación sobre la consistencia comprometida con la práctica. Así, en un primer momento hemos considerado los problemas que podría acarrear el hecho de producir un lenguaje normativo no consistente, y a propósito de ello hemos propuesto una recomendación suficientemente amplia, capaz de alcanzar no sólo a la terminología, sino también a otros aspectos de la redacción. Pensamos que una fórmula bosquejada en estos términos puede evitar aspectos cuestionables que quedarían obviados si se adoptasen fórmulas más restringidas (ceñidas, por ejemplo, a los tecnicismos exclusivamente). Sin embargo, el hecho de tratar de ofrecer una recomendación suficientemente amplia, no marca el final del compromiso con la práctica; y en este sentido cobra especial relevancia la pregunta de si una recomendación como la propuesta ha de seguirse en todo

\footnotetext{
${ }^{38}$ Ley Orgánica No 1/2004..., cit. nota n. 2, pp. 42177, 42188.

${ }^{39}$ Ley Orgánica № $1 / 2004 \ldots$, cit. nota n. 2, p. 42188.

${ }^{40}$ Ley $\mathrm{N}^{\circ}$ 1/1996, de 10 de enero, de Asistencia Jurídica Gratuita, Boletín Oficial del Estado $\mathrm{N}^{\circ} 11$, viernes 12 enero 1996, p. 795.
} 
caso. La respuesta, como ya hemos visto, parece que ha de ser negativa, porque aunque quizá extrañe en un principio, no parece que pueda afirmarse que la recomendación de consistencia sea en todo caso un instrumento idóneo, para lograr determinados objetivos que podrían considerarse desde la eficacia o la efectividad de las normas. Existen casos en los que el hecho de seguir la recomendación de consistencia, podría llevarnos a un estancamiento en el camino hacia la mejora del lenguaje normativo, o bien hacia la imposibilidad de que el Gobierno lleve a cabo determinados objetivos que pueden estar justificados, dentro de los parámetros que conforman el Estado de Derecho. En otros supuestos, el hecho de seguir la recomendación Ileva aparejada la circunstancia de cuestionarla, al tiempo, por diferentes circunstancias. Parece, por tanto, que todos estos aspectos que, en definitiva, son el resultado de un análisis de la consistencia comprometido con el ámbito práctico, deberían estar de algún modo presentes en la recomendación de consistencia. Pero ¿cuál podría ser la fórmula más apropiada para llevar a buen término este propósito? A continuación ofrecemos algunas propuestas.

En un principio, podría pensarse en una recomendación con condiciones, es decir, una recomendación que estableciese una forma de actuar, pero que al mismo tiempo dejase constancia de que dicha forma de actuar no tiene por qué seguirse en determinados supuestos. Ésta es una posibilidad que está encontrando reflejo en numerosos manuales y guías de redacción normativa, a propósito de algunas de las recomendaciones que se consideran en estos documentos $^{41}$. Sin embargo, tal disposición puede resultar cuestionable por varias razones. En este sentido, quizá resulte interesante una apreciación que recoge Florijn en uno de sus trabajos, a propósito de las instrucciones de redacción normativa en los Países Bajos. Partiendo de determinadas inconsistencias entre recomendaciones que pueden diagnosticarse en este documento, el autor citado señala que, probablemente teniendo conciencia de ello, quienes redactaron las instrucciones formularon varias de ellas con sutiles relativizaciones, bien en la propia instrucción o bien en la explicación que la acompaña ${ }^{42}$. Si pretendié-

\footnotetext{
${ }^{41} \mathrm{~A}$ modo de ejemplo, podemos referirnos a la recomendación de utilizar un lenguaje no sexista, que en numerosos manuales de redacción, de un modo u otro, se establece su utilización en lo posible. Vid. LEGISLATIVE REFERENCE SERVICE. ALABAMA, Drafting style manual, [s. e.], [s. I.], [s. d.]. En http://alabamaadministrativecode.state.al.us/docs/instructionmanual.pdf [visto el 08/03/12]; BUREAU OF LEGISLATIVE RESEARCH, Legislative Drafting Manual for the State of Arkansas, State of Arkansas, Arkansas, 2010, p. 43. En http://www.arkleg.state.ar.us/bureau/legal/Publications/Bill\%20Drafting\%20 Manual\%2010-08-06.pdf [visto el 08/03/12]; TAKAYAMA, K, H. (Dir.), Hawaii Legislative Drafting Manual, Legislative Referente Bureau, reprint, Honolulu, 2007, p. 29. En http://hawaii.gov//rb/rpts96/dftman. pdf [visto el 08/03/12].

${ }^{42}$ FloRIJN, Nico, "The Instructions for Legislation in The Nethernands. A Critical Appraisal", Legisprudence. Internacional Journal for the Study of Legislation, Vol. IV, 2, 2010, p. 189.
} 
ramos adaptar tal propuesta a la cuestión que nos toca, podríamos proponer una fórmula en la que se recomendase observar la consistencia en el lenguaje normativo, al menos en aquellos casos en los que fuera posible. No obstante, una propuesta de estas características resultaría demasiado vaga e inconcreta (¿cuándo debe entenderse que no es posible la observancia de la consistencia?). Al final, como señala el autor antes citado, la opción de establecer fórmulas con relativizaciones se convertiría en el origen de numerosas faltas de claridad y calidad en el texto de las instrucciones ${ }^{43}$.

Es cierto, no obstante, que quizá pudieran ensayarse propuestas más concretas en la línea anterior, con la intención de aminorar el grado de penumbra que probablemente aparecería en el caso de que se aplicase la opción precedente. Así, por ejemplo, podría recomendarse observar la consistencia en el lenguaje normativo, siempre que ello no fuera en detrimento de la efectividad de la norma. Sin embargo, esta propuesta tampoco conseguiría aminorar el nivel de oscuridad al que antes nos referíamos, al menos de forma suficientemente satisfactoria. En principio habría que decir que para dejar cumplida constancia del esquema de objeciones expuesto, en cuanto a la posibilidad de considerar una recomendación general, la fórmula en cuestión debería haber sido mucho más extensa. En ella deberían quedar recogidos todos los casos en los que pudiera resultar cuestionable la recomendación. Pero esto no es todo. Si se relativiza la recomendación que nos interesa, en buena lógica habría que seguir el mismo sistema con el resto de recomendaciones. Así, por ejemplo, en el caso de la claridad. Aunque pueda entenderse que ésta ha de preservarse por lo general, también es cierto que en determinados supuestos no será así; es posible, por ejemplo, que el hecho de llegar a un acuerdo político, dé como resultado un texto que no sea todo lo claro que fuera de desear. Circunstancias como ésta invitan a pensar que, también en recomendaciones como ésta, la de claridad, deberían proponerse fórmulas con relativizaciones. Sin duda, esta práctica extendida a buena parte de las recomendaciones de las directrices de técnica normativa, daría como resultado un texto poco claro y no precisamente de fácil manejo. Quizá por todo ello merezca la pena seguir reflexionando acerca de propuestas más sugerentes.

Es cierto que normalmente, en los manuales y guías de redacción normativa se ofrece una lista de recomendaciones que consideran numerosos aspectos del lenguaje. Sin embargo, recientemente Xanthaki ha propuesto un modelo más general, que al menos en parte puede ser compatible con el sistema señalado, y que puede resultar especialmente interesante para el propósito que nos hemos fijado en este trabajo. La propuesta a la que nos referimos establece una serie de

${ }^{43}$ FLORIJN, "The Instructions", cit. nota n. 42, p. 189. 
recomendaciones ordenadas conforme a un sistema de ponderación, que puede contribuir a la claridad en la presentación de las recomendaciones, así como también a su presentación de acuerdo a un orden lógico; aspectos éstos que al menos en una parte considerable quedan obviados, si seguimos la propuesta anterior de ofrecer recomendaciones con relativizaciones ${ }^{44}$. A continuación expondremos los aspectos básicos del planteamiento en cuestión. Antes, no obstante, quisiéramos señalar que detrás del mismo es posible localizar un fundamento más profundo, que sienta sus bases en la importancia que debe reconocerse al Parlamento en materia legislativa, y que viene a reforzar con ello uno de los puntos de apoyo del Estado de Derecho ${ }^{45}$. Como es sabido, uno de sus caracteres generales es la división de poderes, en la que encontramos "lógico predominio" del poder legislativo, por cuanto es poder constituyente, constitucional $y$, una vez constituido, el poder parlamentario que produce las normas ${ }^{46}$. Además de ello, y siguiendo con el Estado de Derecho, debemos también referirnos al Imperio de la ley, que sirve también de fundamento para el planteamiento que vamos a exponer, por cuanto desde él se solicita claridad en el lenguaje normativo ${ }^{47}$.

La propuesta a la que hacemos referencia puede, a grandes rasgos, resumirse como sigue ${ }^{48}$. El principio de efectividad ocupa el lugar más alto en la escala a la que antes nos referíamos. Como ya señalamos, con él se hace referencia a la consecución de los objetivos para los cuales se crea la norma. A continuación localizamos el principio de eficacia, que hace referencia a la correspondencia entre las actitudes y comportamientos de la población y las actitudes y comportamientos prescritos por el legislativo. De este modo la eficacia tendrá lugar cuando la norma se obedezca ${ }^{49}$. Se trata, por tanto, del ajuste de la ciudadanía a la conducta que se establece en la norma ${ }^{50}$. Ciertamente, este aspecto tiene

\footnotetext{
${ }^{44}$ Un ejemplo de ello se considera en FLORIJN, "The Instructions", cit. nota n. 42, p. 189.

45 Zapatero, El Arte, cit. nota n. 20, pp. 367.

${ }^{46}$ Díaz, Elías, "Estado de Derecho y legitimidad democrática", en Estado, justicia, derechos, Alianza Editorial, primera reimpresión, Madrid, 2011, p. 81.

${ }^{47}$ Zapatero, Virgilio, "De la jurisprudencia a la legislación", Doxa, 15-16 (1994), p. 779.

${ }^{48}$ En la exposición de principios seguiremos principalmente las siguientes referencias, aunque matizaremos el discurso con otros trabajos: XANTHAKI, "Drafting", cit. nota n. 18, pp. 114, 115, 116, 120; XanthaKı, Helen, "On Transferability of Legislative Solutions: The Functionality Test", en Drafting Legislation. A Modern Approach, Ashgate, England, 2008, pp. 4, 5, 6, 9, 10, 11-15.

${ }^{49}$ Puede consultarse además Zapatero, El Arte, cit. nota n. 20, p. 33; KarPen, Ulrich, "Improving Democratic Development by Better Regulation", en Drafting Legislation. A Modern Approach, Ashgate, England, 2008, p. 157.

${ }^{50}$ Ansuátegul, Francisco Javier, "El concepto de Derecho", en Curso de Teoría del Derecho, Marcial Pons, Madrid, 2000, p. 27.
} 
una importancia destacada en el ámbito de la teoría del derecho, pero con razón, cada vez se está atribuyendo más relevancia en determinados contextos al principio considerado en primer lugar. Si se pretende guiar el comportamiento de la ciudadanía en un sentido determinado, es porque se entiende que tal circunstancia es el cauce adecuado para poder alcanzar los objetivos que se pretenden con la norma ${ }^{51}$. Si se tiene en cuenta tal detalle, podrá comprobarse que el orden de exposición desarrollado hasta el momento en modo alguno es gratuito.

Siguiendo la progresión en grado descendente encontramos en un mismo nivel los principios de claridad, precisión y no ambigüedad. Con la claridad se hace referencia a la cualidad de un texto de ser claro y fácilmente percibido o entendido. Por otro lado, la precisión es la exactitud de expresión o detalle en la redacción, un principio que sin duda resulta sugerente, pero que si se llega demasiado lejos con él, probablemente dará lugar a redundancias, verbosidad o incluso lagunas jurídicas. En tercer lugar, dentro del nivel que ahora nos ocupa encontramos la ambigüedad, que aparece cuando nos encontramos ante un significado inexacto o incierto. A propósito de la misma podemos considerar diferentes niveles. A continuación nos referimos a dos de ellos. De un lado, la ambigüedad semántica, que tiene lugar cuando nos encontramos con una palabra que puede tener varios significados. De otro lado, podemos considerar la ambigüedad sintáctica, en la que los significados diferentes surgen a consecuencia de la ordenación de las palabras, o de la estructura de la frase que se considere. Suele tener lugar, por ejemplo, a consecuencia del uso cuestionable de los pronombres demostrativos y relativos, o en las frases de condición o excepción ${ }^{52}$.

Finalmente, tenemos en el nivel más bajo los principios de sencillez o lenguaje Ilano y el lenguaje neutral en cuanto al género, que se conciben como una herramienta para la consecución de los principios situados en el nivel inmediatamente superior. Los principios señalados en primer lugar son las solicitudes que se han venido reclamando desde el Plain English, un movimiento al que nos referimos anteriormente, y que en este espacio merece que le dediquemos algunas líneas más. En un sentido muy general, podría decirse que con dicho movimiento se pretende promover la democracia y el Imperio de la ley (rule of law), por medio de una legislación que sea accesible a las personas que conforman la audiencia de dicha legislación ${ }^{53}$. Desde esta perspectiva, por tanto, se propone un uso deliberado de la simplicidad, con vistas

\footnotetext{
51 Zapatero, El Arte, cit. nota n. 20, p. 33.

52 Zapatero, El Arte, cit. nota n. 20, p. 260.

${ }^{53}$ Sullivan, Ruth, "The Promise of Plain Language Drafting", McGill Law Journal, Vol. 47, 2001, p. 97.
} 
a conseguir una comunicación eficaz ${ }^{54}$. Para alcanzar tal objetivo se utilizan varios recursos. Así, por ejemplo, el uso de palabras que entienden las personas sin una formación legal especial. Ello, no obstante, no quiere decir que estemos ante una versión simplificada del lenguaje ${ }^{55}$. Por otra parte, con el lenguaje neutral en cuanto al género (o con la expresión lenguaje no sexista, que utilizamos anteriormente) se solicita hacer visibles a las mujeres en el lenguaje. Esta práctica se viene observando desde hace varios años en determinados contextos, si bien en otros se está empezando a hacer visible en las décadas más recientes ${ }^{56}$.

¿En qué lugar del esquema bosquejado podría ubicarse la cuestión de la consistencia en el lenguaje normativo? A nuestro entender podría quedar enmarcada como recomendación de sencillez o lenguaje llano. Quizá, a este respecto, sea interesante dejar constancia de que en algunas referencias se cuestiona la variación elegante a propósito del lenguaje $\mathrm{llano}^{57}$, o que entre los objetivos de éste se ha considerado la consistencia ${ }^{58}$. A fin de cuentas, estamos estudiando un recurso que puede contribuir a un lenguaje más sencillo. Esta nueva propuesta contribuye a aportar la claridad que faltaba en los intentos precedentes, sin olvidar además el planteamiento que se ha venido bosquejando en este estudio (el hecho de que no parece que podamos hablar de una recomendación universal). Ello así queda confirmado, si se tiene en cuenta que la disposición de principios que consideramos anteriormente no es gratuita, dado que con ella, además de conseguirse una exposición más ordenada, se establecen criterios de prelación en caso de posibles enfrentamientos entre principios o recomendaciones. Esta circunstancia, no obstante, ya se ha contemplado de algún modo en el ámbito de la teoría de la legislación, a propósito de las posibles tensiones que pueden darse, por ejemplo, entre la racionalidad lingüística (en la que podrían encuadrarse los dos primeros niveles del planteamiento expuesto en sentido ascendente) y la racionalidad pragmática (que hace referencia a la eficacia de la norma). Ante enfrentamientos de este tipo se ha dicho que las exigencias de

\footnotetext{
${ }^{54}$ Salembier, Paul, Legal and Legislative Drafting, LexisNexis, Canada, 2009, p. 409.

${ }^{55}$ Macdonald, Ros, "Writing Better Decisions: Plain English in Decision Writing", en Legal Language and the Search for Clarity, Peter Lang, Bern, 2006, pp. 333 y 334.

${ }^{56}$ Como ya se señaló, la trayectoria que se está siguiendo puede consultarse en Centenera SánCHEz-SECO, Nombradas, cit. nota n. 36. Para su desarrollo en otros contextos puede verse Centenera SánCHez-SeCo, Fernando, "Desdoblamientos, sustantivos genéricos y otros recursos perceptibles en el ordenamiento jurídico español: ¿una moda de hoy o un cambio con mañana?", Universitas. Revista de Filosofía, Derecho y Política, № 11, 2010, pp. 71-73. En http://universitas.idhbc.es/n11/11-05.pdf [visto el 06/06/12].

${ }^{57}$ Asprey, Michele M., Plain Language for Lawyers, The Federation Press, Sydney, 2003, pp. 129 y 130.

${ }^{58}$ Macdonald, Ros, "Writing Better", cit. nota n. 55, p. 334.
} 
la racionalidad lingüística deberían subordinarse a aquellas que pertenecen a otros tipos de racionalidad, como el considerado en segundo lugar ${ }^{59}$.

Desde una perspectiva más concreta, el desarrollo expuesto podría plantearse como sigue. Demos por hecho que la recomendación de consistencia ha quedado ubicada en el principio de lenguaje Ilano. En tal circunstancia, en principio el equipo de redacción debería atender a la recomendación de consistencia, pero ésta quedaría cuestionada en el caso de que entrase en tensión con otros principios situados en un nivel igual o mayor. Así, por ejemplo, acontecería, si el hecho de mantener la consistencia en el lenguaje normativo fuera un obstáculo para lograr la claridad (en cuyo caso el enfrentamiento se dirimiría a favor de este último principio), o para que las normas se cumplan por parte de la audiencia a la que van destinadas (supuesto en el que prevalecería el principio de eficacia, que es superior a aquel en el que encuadramos la recomendación de consistencia). Del mismo modo acontecería en el caso de que la consistencia se enfrentase a los objetivos que se pretenden con la publicación de la norma. En tal supuesto prevalecerían estos últimos, dado que, de nuevo, el principio de efectividad es superior a aquel en el que hemos encuadrado la recomendación de consistencia.

\section{BibliografíA}

Ansuátegui, Francisco Javier, "El concepto de Derecho", en Curso de Teoría del Derecho, Marcial Pons, Madrid, 2000.

Aristóteles, Ética a Nicómaco, Centro de Estudios Políticos y Constitucionales, Madrid, 1999.

Asprey, Michele M., Plain Language for Lawyers, The Federation Press, Sydney, 2003.

Atienza, Manuel, Contribución a una teoría de la legislación, Civitas, Madrid, 1997.

Azuelos-Atias, Sol, "On the incoherence of Legal Language to the General Public", International Journal for the Semiotics of Law, June, 2010. En http:// www.springerlink.com/content/d072m75556167j35/ [visto el 16/08/10].

BeNION, Francis, On Statute Law, Logman, [s. I.], 1990. En http://www.francisbennion.com/pdfs/fb/1990/1990-002-187-statute-law-pt2-ch12.pdf [visto el 16/08/10].

Bentham, Jeremy, Nomografía o el arte de redactar leyes, Centro de Estudios Políticos y Constitucionales, Madrid, 2004.

${ }^{59}$ Sobre estas cuestiones puede consultarse Zapatero, El Arte, cit. nota n. 20, pp. 265 y ss.; AtienZA, Manuel, Contribución a una teoría de la legislación, Civitas, Madrid, 1997, pp. 58 y 59; Oliver-Lalana, A. Daniel, "Los argumentos de eficacia en el discurso parlamentario", Doxa, 31 (2008), p. 539. 
BUREAU OF LEGISLATIVE RESEARCH, Legislative Drafting Manual for the State of Arkansas, State of Arkansas, Arkansas, 2010. En http://www.arkleg.state. ar.us/bureau/legal/Publications/Bill\%20Drafting\%20Manual\%2010-08-06. pdf [visto el 08/03/12].

ButT, Peter and CASTLE, Richard, Modern Legal Drafting. A Guide to Using Clearer Language, Cambridge University Press, Cambridge, 2001.

Cazorla Prieto, Luis María, El Lenguaje Jurídico Actual, Aranzadi, Pamplona, 2007.

Centenera Sánchez-Seco, Fernando, "Desdoblamientos, sustantivos genéricos y otros recursos perceptibles en el ordenamiento jurídico español: ¿una moda de hoy o un cambio con mañana?", Universitas. Revista de Filosofía, Derecho y Política № 11, 2010. En http://universitas.idhbc.es/n11/11-05. pdf [visto el 06/06/12].

_ Nombradas. También en el ámbito jurídico, Dykinson, Madrid, 2010.

Centenera Sánchez-Seco, Fernando, "Una aproximación a la aplicación parcial del lenguaje neutral en la norma", Cuadernos Electrónicos de Filosofía del Derecho $\mathrm{N}^{\circ} 18,2009$. En http://ojs.uv.es/index.php/CEFD/article/view/129/141 [visto el 10/04/12].

CHRISTOPHeR, Williams, "The End of the 'Masculine Rule'? Gender-Neutral Legislative Drafting in the United Kingdom and Ireland", Statute Law Review, 29 (3).

CONNATSER, Bradford R., "Two centuries in progress in technical communication", Technical writing and communication Vol. 37 (2), 2007.

Crabbe, Vcrac, "The ethics of legislative drafting", Commonwealt law bulletin, Vol. 36, 1, March 2010.

Díaz, Elías, "Estado de Derecho y legitimidad democrática", en Estado, justicia, derechos, Alianza Editorial, primera reimpresión, Madrid, 2011.

DICKERSON, Reed, The Fundamentals of Legal Drafting, Little, Brown and Company, Boston 1986.

FloRIJN, Nico, "The Instructions for Legislation in The Nethernands. A Critical Appraisal", Legisprudence. Internacional Journal for the Study of Legislation, Vol. IV, 2, 2010.

FUNDACIÓN HUMANISMO POLÍTICO A. C., Manual de Técnica Legislativa, [s. e.], [s. I.], [s. d.]. En http://www.agora-parl.org/sites/default/files/FHP\%20 -\%20Manual\%20de\%20Tecnica\%20legislativa\%20-\%20ES\%20-\%20PI.pdf [visto el 08/03/12].

Galiana Saura, Ángeles, La legislación en el Estado de Derecho, Dykinson, Madrid, 2003. 
GRUPPO DI LAVORO PER LA REVISIONE DEL MANUALE REGIONALE DI DRAFTING, Regole e suggerimenti per la redazione dei testi normativi, G. Tamara Tarabusi e Massimo Signorile del Consiglio regionale della Toscana, Terza edizione, Toscana, 2007. En: http://lexview-int.regione.fvg.it/iterleggi/ drafting/drafting.pdf [visto el 08/03/12].

INFORME DE LA COMISIÓN DE MODERNIZACIÓN DEL LENGUAJEJURÍDICO, 2011. En: http://portal.uned.es/pls/portal/docs/PAGE/UNED_MAIN/LAUNIVERSIDAD/VICERRECTORADOS/SECRETARIA/NORMATIVA/RECOMENDACIONES_DE_LA_COMISI\%C3\%93N_DE_MODERNIZACI\%C3\%93N_ DEL_LENGUAJE_JUR\%C3\%8DDICO.PDF [visto el 23/01/12].

KARPEN, Ulrich, "Improving Democratic Development by Better Regulation", en Drafting Legislation. A Modern Approach, Ashgate, England, 2008.

KelLy, David, "The victorian experience of plain drafting", en Legislation and its interpretation. Discussion and seminar papers, Law Commission, Wellington, 1988.

LAPORTA, Francisco J., "Imperio de la ley y seguridad jurídica", en Estado, justicia, derechos, Alianza Editorial, primera reimpresión, Madrid, 2011.

Laws CB, Stephen, "Drawing the Line", en Drafting Legislation. A Modern Approach, Ashgate, England, 2008.

LEGISLATIVE REFERENCE SERVICE. ALABAMA, Drafting style manual, [s. e.], [s. I.], [s. d.]. En http://alabamaadministrativecode.state.al.us/docs/instruction manual.pdf [visto el 08/03/12].

Ley 1/1996, de 10 de enero, de asistencia jurídica gratuita, Boletín Oficial del Estado $\mathrm{N}^{0}$ 11, viernes 12 enero 1996.

Ley 20/2007, de 11 de julio, del Estatuto del trabajo autónomo, Boletín Oficial del Estado $N^{\circ} 166$, jueves 12 julio 2007.

Ley Orgánica 1/2004, de 28 de diciembre, de Medidas de Protección Integral contra la Violencia de Género, Boletín Oficial del Estado № 313, miércoles 29 diciembre 2004.

López Ruiz, Miguel, Redacción Legislativa, Senado de la República, México, 2002. En http://www.senado.gob.mx/biblioteca/content/catalogos/b_digital/ bibliodigital/redaccionlegislativa.pdf [visto el 19/07/10].

MaCDOnald, Ros, "Writing Better Decisions: Plain English in Decision Writing", en Legal Language and the Search for Clarity, Peter Lang, Bern, 2006.

Marcilla Córdoba, Gema, Racionalidad legislativa. Crisis de la ley y nueva ciencia de la legislación, Centro de Estudios Constitucionales, Madrid, 2005.

Martino, Antonio, Manual de Técnica Legislativa, [s. e.], [s. I.], [s. d.]. En http:// exwww.dsp.unipi.it/didattica/Digesto/manual.html [visitado el 01/12/11].

Meseguer YeBRA, Joaquín, Guía práctica para la elaboración de textos normativos. Directrices estatales de técnica normativa, Boch, Barcelona, 2008. 
Miers, David R. and PAGe, Alan C., Legislation, Sweet \& Maxwell, London, 1982.

Oliver-Lalana, A. Daniel, "Los argumentos de eficacia en el discurso parlamentario", Doxa, 31 (2008).

Peces-Barba Martínez, Gregorio, Diez lecciones sobre Ética, Poder y Derecho, Dykinson, Madrid, 2010.

Prieto De Pedro, Jesús, Lenguas, lenguaje y derecho, Civitas, Madrid, 1991.

REAL ACADEMIA ESPAÑOLA, Diccionario de la Lengua Española, [s. e.], [s. I.], 2001. En: http://buscon.rae.es/drael/ [visitado el 02/01/12].

Resolución de 28 de julio de 2005, de la Subsecretaría, por la que se da publicidad al Acuerdo del Consejo de Ministros, de 22 de julio de 2005, por el que se aprueban las Directrices de técnica normativa, Boletín Oficial del Estado No 180, viernes 29 julio 2005.

Sáinz Moreno, Fernando, "Problemas actuales de técnica normativa", Anuario Jurídico de La Rioja № 1, 1995.

Salembier, Paul, Legal and Legislative Drafting, LexisNexis, Canada, 2009.

Seidman, Ann, Seidman, Robert B., Abeyesekere, Nalin, Legislative Drafting for Democratic Social Change. A Manual for Drafters, Kluwer Law International, London, 2001.

Strunk, William y White, E. B., The elements of style, Allyn and Bacon, third edition, Boston, 1979.

Suluivan, Ruth, "The Promise of Plain Language Drafting", McGill Law Journal, Vol. 47, 2001.

TакAуAмA, K, H. (Dir.), Hawaii Legislative Drafting Manual, Legislative Referente Bureau, reprint, Honolulu, 2007. En http://hawaii.gov//rb/rpts96/dftman.pdf [visto el 08/03/12].

TEXAS LEGISLATIVE COUNCIL, Drafting Manual, [s. e.], [s. I.], 2011. En http:// www.tlc.state.tx.us/legal/dm/sec723.htm [visitado el 08/03/12].

WRóbleWSKI, Jerzy, Constitución y teoría general de la interpretación jurídica, Civitas, Madrid, 1985.

XaNTHAKI, Helen, "Drafting manuals and quality in legislation: positive contribution towards certainty in the law or impediment to the necessity for dynamism of rules?", Legisprudence. International Journal for the Study of Legislation, Vol. IV, 2, 2010.

, "On Transferability of Legislative Solutions: The Functionality Test", en Drafting Legislation. A Modern Approach, Ashgate, England, 2008.

ZaPATERO, Virgilio, "De la jurisprudencia a la legislación", Doxa, 15-16 (1994). - El Arte de Legislar, Aranzadi, Pamplona, 2009. 